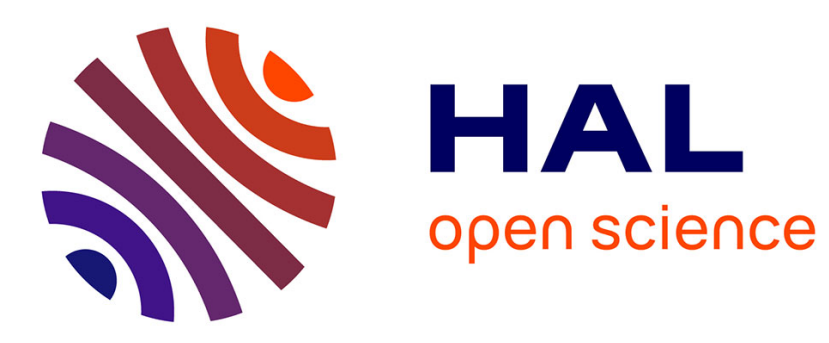

\title{
Vibration-based monitoring of civil structures with subspace-based damage detection
}

\author{
Michael Döhler, Falk Hille, Laurent Mevel
}

\section{To cite this version:}

Michael Döhler, Falk Hille, Laurent Mevel. Vibration-based monitoring of civil structures with subspace-based damage detection. Erika Ottaviano, Assunta Pelliccio, Vincenzo Gattulli. Mechatronics for Cultural Heritage and Civil Engineering, 92, Springer, pp.307-326 2018, Intelligent Systems, Control and Automation: Science and Engineering, 10.1007/978-3-319-68646-2_14 . hal-01684072

\section{HAL Id: hal-01684072 \\ https://hal.inria.fr/hal-01684072}

Submitted on 15 Jan 2018

HAL is a multi-disciplinary open access archive for the deposit and dissemination of scientific research documents, whether they are published or not. The documents may come from teaching and research institutions in France or abroad, or from public or private research centers.
L'archive ouverte pluridisciplinaire HAL, est destinée au dépôt et à la diffusion de documents scientifiques de niveau recherche, publiés ou non, émanant des établissements d'enseignement et de recherche français ou étrangers, des laboratoires publics ou privés. 


\title{
Vibration-based monitoring of civil structures with subspace-based damage detection
}

\author{
Michael Döhler, Falk Hille and Laurent Mevel
}

\begin{abstract}
Automatic vibration-based structural health monitoring has been recognized as a useful alternative or addition to visual inspections or local non-destructive testing performed manually. It is, in particular, suitable for mechanical and aeronautical structures as well as on civil structures, including cultural heritage sites. The main challenge is to provide a robust damage diagnosis from the recorded vibration measurements, for which statistical signal processing methods are required. In this chapter, a damage detection method is presented that compares vibration measurements from the current system to a reference state in a hypothesis test, where datarelated uncertainties are taken into account. The computation of the test statistic on new measurements is straightforward and does not require a separate modal identification. The performance of the method is firstly shown on a steel frame structure in a laboratory experiment. Secondly, the application on real measurements on S101 Bridge is shown during a progressive damage test, where damage was successfully detected for different damage scenarios.
\end{abstract}

Key words: Subspace methods, damage detection, statistical tests, vibrations, structural health monitoring.

\section{Introduction}

Structural health monitoring (SHM) has the goal of determining the system health, i.e. the occurrence and characterization of damages, based on the measurement data

Michael Döhler, Laurent Mevel

Inria, I4S / IFSTTAR, COSYS, SII, Campus universitaire de Beaulieu, 35042 Rennes, France, email: michael.doehler@inria.fr, laurent.mevel@inria.fr

Falk Hille

BAM Federal Institute of Materials Research and Testing, Unter den Eichen 87, 12205 Berlin, Germany, e-mail: falk.hille@bam.de 
recorded on the monitored structure [16]. With statistical signal processing techniques, one can find damages automatically within a monitored structure, before they grow to dangerous extents. Automatic inspection is of enormous value for structures with high failure consequences or poor accessibility, such as bridges, structures of cultural heritage, offshore constructions, large engineering structures, power plants, etc. With these means, maintenance operations can be optimized and costly downtime kept to a minimum, while the structural safety is increased.

Vibration-based SHM has shown great potential for automated damage monitoring [8]. Methods for damage assessment are categorized into four groups of increasing difficulty and complexity: 1/ detection, 2 / localization, 3/ quantification, and 4/ prognosis of remaining life [21]. Many methods have been proposed in the literature (see e.g. reviews [9] and [15]), with however few methods taking into account the statistical nature of the vibration measurements, which is essential for robustness under realistic (noisy) operational conditions in practice. Among the methods for damage assessment, the detection methods are currently the most developed and some of them have matured to an industrial application level [22].

Vibration-based damage detection is performed on the rationale that damage affects the stiffness of the monitored structure and, thus, changes its dynamic properties. The analysis of the dynamic properties can be performed entirely based on measurements, without the need of a finite element model. Many of the currently used vibration-based methods for early damage detection perform modal system identification and compare the obtained modal parameter of the actual state with those of the undamaged state, e.g. by using control charts [19, 20]. In this context, especially the natural frequencies are used for a comparison, as they can be reliably identified. However, the automated estimation of modal parameters from a system identification method and matching them from measurements of different states of the structure for their comparison might require an extensive preprocessing step. Other methods avoid the system identification step in the possibly damaged state. For instance, such methods include non-parametric change detection based on novelty detection [24, 25], whiteness tests on Kalman filter innovations [7] or other data-driven features that are sensitive to changes in the modal parameters.

The subspace-based damage detection approach $[4,6,11,12,13]$, which will be considered in this chapter, also falls into the latter category. In this method, a data-driven model is obtained in the reference state and compared to measurements from an unknown, possibly damaged state. This comparison is performed using a subspace-based residual function and a $\chi^{2}$-test built on it for a hypothesis test, without actually estimating the modal parameters in the unknown, possibly damaged states. In this way, the entire structural response is taken into account. This approach provides a complete statistical framework for analyzing a damage residual for both reference and possibly damaged structures. The asymptotic probability distribution of the considered damage residual is given and a statistical hypothesis test to detect damage in the structure has been proposed.

In this chapter, the subspace-based damage detection method is presented. The performance of the method is firstly shown on a steel frame structure in a laboratory experiment. Secondly, the application on real measurements on S101 Bridge is 
shown during a progressive damage test, where damage was successfully detected for different damage scenarios.

\section{Subspace-based damage detection}

In this section, the subspace-based damage detection algorithm is introduced. With this global automated damage detection method, vibration measurements from the current system are compared to a reference state in a subspace-based residual vector. In a hypothesis test, the uncertainties of the residual are taken into account and the respective test statistic is compared to a threshold in order to decide if the structure is damaged or not.

\subsection{Dynamic structural system model}

The vibration behaviour of the monitored structure is assumed to be described by a linear time-invariant dynamical system

$$
\mathscr{M} \ddot{z}(t)+\mathscr{C} \dot{z}(t)+\mathscr{K} z(t)=v(t)
$$

where $t$ denotes continuous time; $\mathscr{M}, \mathscr{C}, \mathscr{K} \in \mathbb{R}^{m \times m}$ are mass, damping, and stiffness matrices, respectively; the state vector $z(t) \in \mathbb{R}^{m}$ is the displacement vector of the $m$ degrees of freedom of the structure; and $v(t)$ is the external force. Since the excitation is usually ambient and unmeasured for long-term monitoring, the external force is modelled as white noise.

Observed at $r$ sensor positions (e.g. by displacement, velocity or acceleration sensors) at discrete time instants $t=k \tau$ (with sampling rate $1 / \tau$ ), system (1) can be transformed into a discrete-time state space system model [18]

$$
\left\{\begin{aligned}
x_{k+1} & =A x_{k}+v_{k} \\
y_{k} & =C x_{k}+w_{k}
\end{aligned}\right.
$$

with the state vector $x_{k}=\left[z(k \tau)^{T} \dot{z}(k \tau)^{T}\right]^{T} \in \mathbb{R}^{n}$, the measured outputs $y_{k} \in \mathbb{R}^{r}$, the state transition matrix

$$
A=\exp \left(\left[\begin{array}{cc}
0 & I \\
-\mathscr{M}^{-1} \mathscr{K}-\mathscr{M}^{-1} \mathscr{C}
\end{array}\right] \tau\right) \in \mathbb{R}^{n \times n}
$$

and the observation matrix

$$
C=\left[L_{d}-L_{a} \mathscr{M}^{-1} \mathscr{K} \quad L_{v}-L_{a} \mathscr{M}^{-1} \mathscr{C}\right] \in \mathbb{R}^{r \times n}
$$


where $n=2 m$ is the model order and $L_{d}, L_{v}, L_{c} \in\{0,1\}^{r \times m}$ are selection matrices indicating the positions of displacement, velocity or acceleration sensors at the degrees of freedom of the structure, respectively. The state noise $v_{k}$ and output noise $w_{k}$ are unmeasured and assumed to be centered and square integrable.

Damages in the monitored system correspond to changes in the matrices $\mathscr{M}, \mathscr{C}$ or $\mathscr{K}$ in (1), e.g. loss of mass or loss of stiffness, and affect the system matrices $(A, C)$ in (2) and the modal parameters (natural frequencies, damping ratios, mode shapes). Hence, damages in model (1) can be equivalently detected as changes in the system matrices in (2). Based on measurements $y_{k}$ of the monitored system from a reference and from a possibly damaged state, a residual vector is defined based on subspace properties, as outlined in the following section.

\subsection{Description of the method}

In $[4,6]$ a residual vector was proposed whose mean is zero in the reference state, and non-zero in the damaged state. It is computed from the measurements $y_{k}$ without actually identifying the modal parameters in the unknown, possibly damaged state. The considered residual is associated with a covariance-driven output-only subspace identification algorithm as follows.

Define the block Hankel matrix $\mathscr{H}_{p+1, q}$ of the output covariances $R_{i}=\mathbf{E}\left(y_{k} y_{k-i}^{T}\right)$ as

$$
\mathscr{H}_{p+1, q} \stackrel{\text { def }}{=}\left[\begin{array}{cccc}
R_{1} & R_{2} & \ldots & R_{q} \\
R_{2} & R_{3} & \ldots & R_{q+1} \\
\vdots & \vdots & \ddots & \vdots \\
R_{p+1} & R_{p+2} & \ldots & R_{p+q}
\end{array}\right] \stackrel{\text { def }}{=} \operatorname{Hank}\left(R_{i}\right),
$$

where the parameters $p$ and $q$ are chosen such that $\min \{p r, q r\}>n$ with usually $p+1=q$. It possesses the well-known factorization property

$$
\mathscr{H}_{p+1, q}=\mathscr{O}_{p+1} \mathscr{C}_{q}
$$

into the matrices of observability and controllability

$$
\mathscr{O}_{p+1}=\left[\begin{array}{c}
C \\
C A \\
\vdots \\
C A^{p}
\end{array}\right], \quad \mathscr{C}_{q}=\left[\begin{array}{lllll}
G & A G & \ldots & A^{q-1} G
\end{array}\right],
$$

where $G=\mathbf{E}\left(x_{k+1} y_{k}^{T}\right)$ is the cross-covariance between the states and the outputs. From a singular value decomposition of $\mathscr{H}_{p+1, q}$, the left null space matrix $S$ can be obtained with the characteristic property

$$
S^{T} \mathscr{H}_{p+1, q}=0
$$


in the reference state of the structure. If the system is damaged, the matrices $(A, C)$ and thus $\mathscr{H}_{p+1, q}$ change, thus the product $S^{T} \mathscr{H}_{p+1, q}$ is not zero anymore in the damaged state.

Based on these properties, a residual vector is defined as follows. From a set of output measurements $y_{k}, k=1, \ldots, N$, estimates of the output covariances and the Hankel matrix are computed as

$$
\widehat{R}_{i}=\frac{1}{N} \sum_{k=1}^{N} y_{k} y_{k-i}^{T}, \quad \widehat{\mathscr{H}}_{p+1, q}=\operatorname{Hank}\left(\widehat{R}_{i}\right)
$$

and the residual vector is defined as

$$
\zeta=\sqrt{N} \operatorname{vec}\left(S^{T} \widehat{\mathscr{H}}_{p+1, q}\right),
$$

where $\operatorname{vec}(\cdot)$ denotes the column stacking vectorization operator. Due to property (5), the mean of $\zeta$ is zero in the reference state of the structure, and non-zero in the damaged state. The residual vector $\zeta$ is asymptotically Gaussian distributed [4]. Let $\Sigma$ be the asymptotic residual covariance matrix. Then, the corresponding hypothesis test for a decision between $\mathbf{H}_{0}$ : the system is in the reference state, and $\mathbf{H}_{1}$ : the system is in a damaged state, leads to the test statistic

$$
s=\zeta^{T} \Sigma^{-1} \zeta
$$

which is asymptotically $\chi^{2}$ distributed with a non-centrality parameter in the damaged state. To decide if the monitored structure is damaged or not, the test statistic $s$ is compared to a threshold $t$, and damage is announced if $s>t$. The threshold $t$ is typically chosen such that the probability of false alarms (type I error) is below a certain level. An example of the probability distributions of the test statistic $s$ in the reference and damaged states is given in Figure 1. Computational details for obtaining estimates of the null space matrix $S$, covariance matrix $\Sigma$ and the threshold $t$ in the reference state of the monitored structure, which are required for the damage detection test, are given in the Appendix.

Note that the multiplication with $\sqrt{N}$ in the definition of the residual vector (6) ensures its asymptotic Gaussianity through the central limit theorem, and thus the definition of a $\chi^{2}$-distributed test statistic in (7). Moreover, this statistical framework allows the detection of very small damages if the number of measurements $N$ is large enough. A detailed description of the statistical framework and more theoretical insight of this method is given in $[4,12,13,14]$, including parametric test variants.

\subsection{Robustness to environmental nuisances}

In the previous section, the subspace-based damage detection test is given in its most basic version. The test is set up entirely on measurement data without the need of a numerical model, and no modal analysis is required at all. It takes into account 


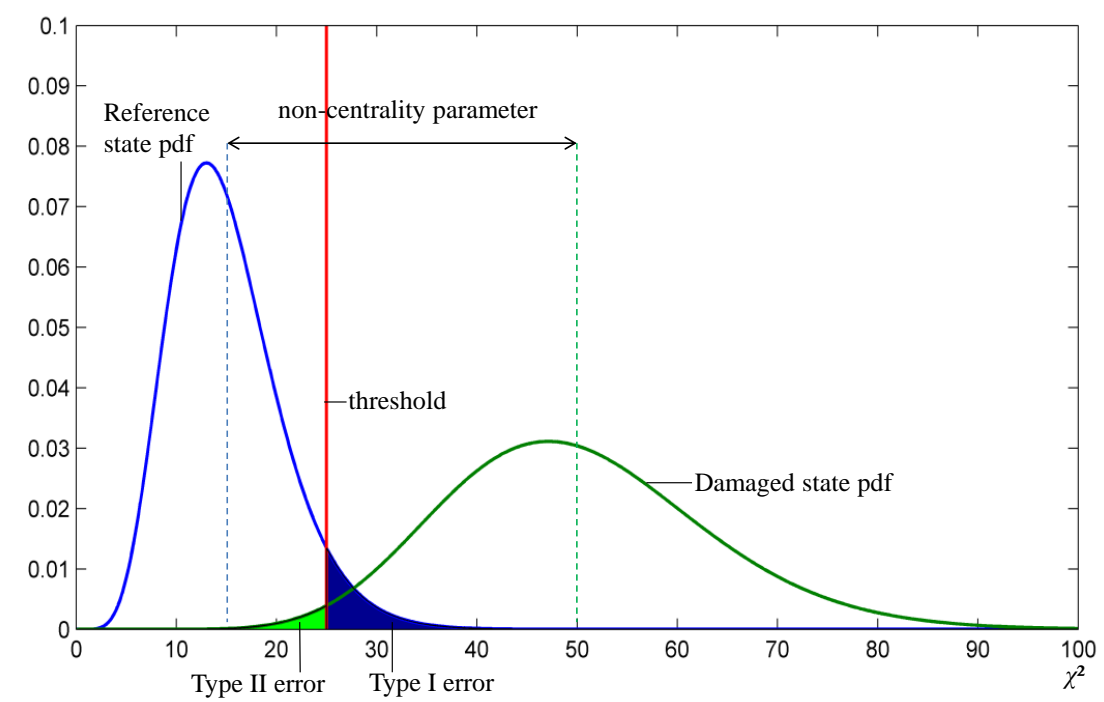

Fig. 1 Scheme of the probability density functions (pdf) of the $\chi^{2}$-distributed damage detection test statistic $s$ in the reference and in a damaged state.

the natural variability that is related to the measurements, which is due to unknown ambient excitation and measurement noise. While it is convenient for many applications, additional robustness to natural environmental changes like excitation or temperature variations can be easily accounted for.

Changes in the ambient excitation properties do not affect the dynamic properties of the structure, but may have an influence on the test statistic (7) that is directly computed on the measurements. Robustness to such changes is achieved by a slight modification of the residual vector (6), where the Hankel matrix estimate $\widehat{\mathscr{H}}_{p+1, q}$ is replaced by the matrix $U_{1}$ of its principal left singular vectors as in (9), leading to the robust residual

$$
\tilde{\zeta}=\sqrt{N} \operatorname{vec}\left(S^{T} U_{1}\right)
$$

Since the principal left singular vectors of $\mathscr{H}_{p+1, q}$ also satisfy the characteristic property (5) of the reference state, the robust residual $\tilde{\zeta}$ similarly detects changes in the dynamic system properties, with the advantage that $U_{1}$ is an orthonormal matrix and thus independent from scaling due to excitation changes. The respective test statistic with the appropriate covariance matrix $\tilde{\Sigma}$ is, analoguously,

$$
\tilde{s}=\tilde{\zeta}^{T} \tilde{\Sigma}^{-1} \tilde{\zeta} .
$$

Further theoretical and implementation details are given in $[12,13]$.

Temperature variations can be taken into account in a non-parametric or in a parametric way. In the non-parametric way, the left null space matrix $S$ and the 
residual covariance matrix $\Sigma$ are computed on several datasets that are recorded under different temperature scenarios [1]. In this way, the variation of the residual due to temperature changes is eliminated in the test statistic. In the parametric way, a model describes the influence of the temperature on the monitored structure. Then, variations in the temperature parameter can be rejected in the test statistic as nuisance or the left null space matrix $S$ can be adjusted with the temperature $[2,5]$.

\section{Application to a steel frame}

In this section, the damage detection method is applied to a scaled two-dimensional section of a jacket-type support structure of an offshore wind turbine in a laboratory experiment. In practice, these support structures are used in deep sea waters of $30 \mathrm{~m}$ and more. They generally include a significant number of highly stressed joints and, therefore, possess a high vulnerability to fatigue damage. In addition, most of the structural components are situated under sea level which makes damage investigation costly. This leads to the application of robust SHM techniques.

\subsection{Laboratory test configuration}

The developed model structure out of steel pipe components represents a scaled twodimensional section of a jacket-type support structure of an offshore wind turbine as they are common for wind park installations. The scale of the general dimensions is approximately 1:10. The lab model is designed with one and a half diagonal bracings between two legs. The upper completion is formed by an I-sectional girder. At the lower end the legs end in steel foot plates. With exception of the damage sections all structural parts of the model are welded. The foot plates are screwed via a transition piece to the lab floor. In the third direction the structure is held on the head girder by an auxiliary construction, whose two fork-like support bars allow for the horizontal movement of the head girder. Figure 2 shows the laboratory test structure in the proof testing facilities at BAM.

The damage to be modeled is supposed to represent fatigue cracks according to the characteristic cyclic stressing which can even escalate by resonance effects as well as due to the notches in the material from the welding process. The descriptiveness of the damage in location and quantification for comparison of different test series within the study as well as reversibility of the inserted damage for the ability to repeat test series were considered for the damage modeling.

For the difficulty in designing a reversible damage in the joint area of a gusset, the damage areas of the laboratory structure were constructed with a shift into the steel tube domain. A crack-like damage can be induced artificially by the loosening of bolted flange connection at one K-type gusset of the model structure. Four of such flange connections are designed, each at the four ends of the K-type gusset. 


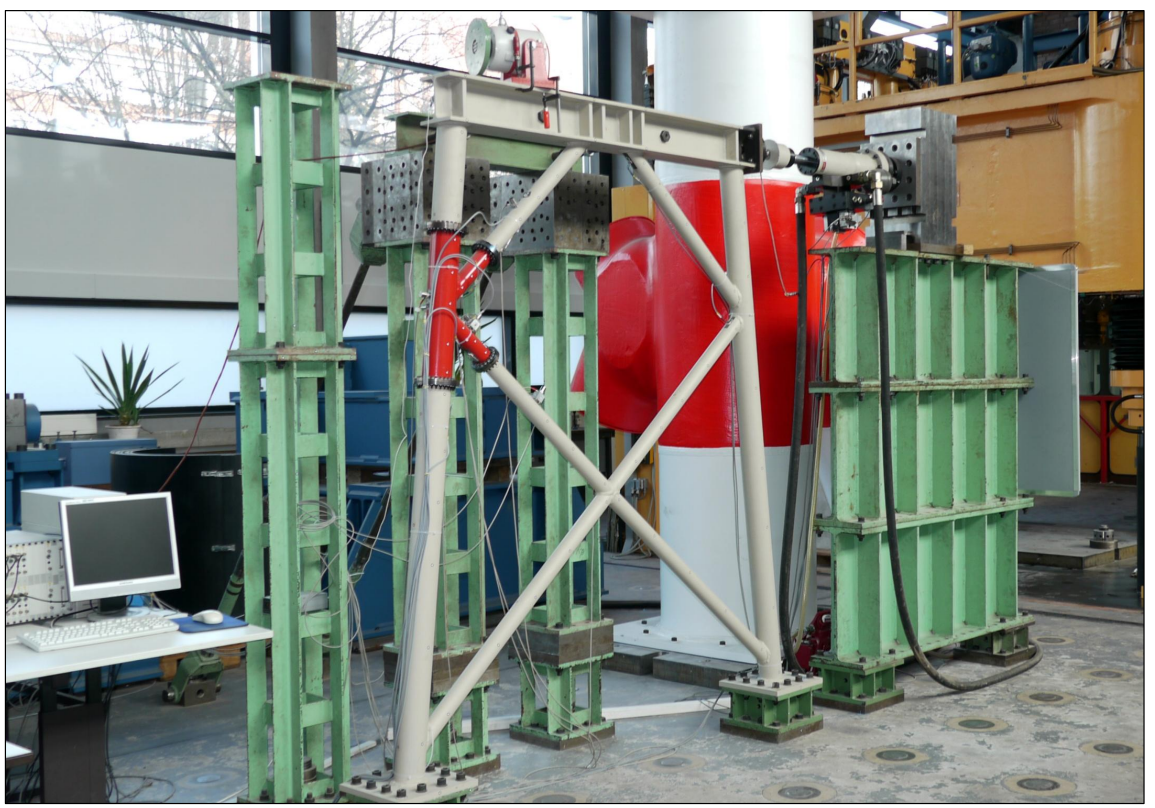

Fig. 2 Laboratory test stand at BAM.

So there were two damage locations at the leg and two at the bracing diagonal. In Figure 2 the four flanges at the end of the red drawn component on the left side of the model can be seen at each end of the red K-type gusset.

During the test series, dynamic excitations and cyclic loading of the laboratory structure were deployed. With different excitations the dependency of the detection of simulated damage on the frequency and energy input of the excitation source was examined. The excitation for the tests was provided by an electrodynamic shaker. A broadband random acceleration signal with a frequency content between $10 \mathrm{~Hz}$ and $1000 \mathrm{~Hz}$ was produced by a shaker control unit and was induced after amplification via the shaker at the top of the structure. The shaker was equipped with a $5 \mathrm{~kg}$ excitation mass and the excitation direction was horizontal and approximately $30^{\circ}$ rotated out of the in-plane-direction. Eight piezoelectric accelerometers were applied at the structure for the vibration measurements. The sensors were connected with the structure by permanent magnets and measured in the direction perpendicular to the surface they were applied to. The signals were amplified, recorded and stored with a 20-channel DIFA measurement unit. 


\subsection{Artificial damage detection tests}

In a first test stage, the general reaction of the test as well as its robustness to changing ambient excitation levels were investigated. The damage location was chosen at the lower brace of the flange. In the reference state all bolts were screwed tight. For inducing damage, successively one, two, three, five or seven adjacent bolts were unscrewed, each representing a fatigue crack of increasing length. Thereby, the loosening of three bolts is comparable with a reduction of the moment of inertia by $3 \%$, whereas the unscrewing of seven bolts means a loss of $30 \%$ of the bending stiffness.

To validate the damage detection algorithm under changing excitation, three excitation (power) levels were used for each damage state. Besides the full scale level, a reduction of $5 \mathrm{~dB}$ and a reduction of $10 \mathrm{~dB}$ with respect to the full scale level were performed and are denoted as "Excitation 1", "Excitation 2" and "Excitation 3" in the tests, respectively. A reduction of $5 \mathrm{~dB}$ accounts for a power ratio of $\approx 0.31$ and an amplitude ratio of $\approx 0.56$ and a reduction of $10 \mathrm{~dB}$ for a power ratio of 0.1 and an amplitude ratio of $\approx 0.31$. The full-scale excitation power level was chosen preliminary in dependence of the shaker performance. For each damage level and each excitation level, four signals of $16.4 \mathrm{~s}$ length was measured.

In Fig. 3(a), the test statistic $s$ from (7) is computed on the datasets with excitation 1 . The test values increase with the damage size, where the damage cases of five and seven loosened bolts are well above the threshold that was established in the reference state. The robustness to unknown changing excitation properties is tested with the robust test variant (8). Results for the three different excitation levels are shown in Figure 3(b). There are small fluctuations of the test values in the reference state and in the states with small damage, but these fluctuations are not significant with respect to the established threshold. For the damages of three or more loosened bolts, these variations seem to be independent from the excitation level, which validates the robustness of the test to changing excitation. The relatively small damage

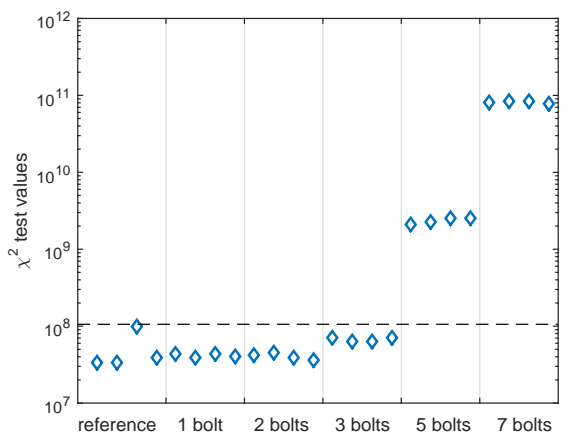

(a) Test (7) under constant excitation levels.

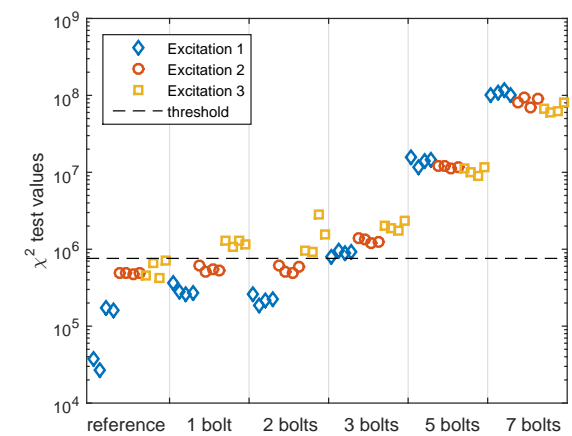

(b) Robust test variant (8) under different excitation levels.

Fig. 3 Damage detection tests on steel frame structure for different damage extents (log-scale). 
of only three loosened bolts can be detected, and the further damages of five and seven loosened bolts are clearly above the threshold. Further details and results of this experiment are given in [10].

\subsection{Fatigue damage detection tests}

In the next step, a fatigue test was accomplished to test the applicability of the damage detection method to real damage. The test procedure consisted of two alternating tasks: the insertion of the fatigue relevant load cycles and the measurement of dynamic response series for determination of the damage detection test value. A load series with a constant alternating horizontal force of $\pm 50 \mathrm{kN}$ was applied at the top girder of the structure with a frequency of $3 \mathrm{~Hz}$. After completion of each load series, a damage test was conducted, where a broadband random excitation signal with a frequency content between $10 \mathrm{~Hz}$ and $1000 \mathrm{~Hz}$ was produced by the shaker. The vibration response was measured with nine piezoelectric accelerometers at the structure. A total of ten load series (with varying number of load cycles) and, therefore, ten damage states were applied. The fatigue damage did not occur at the expected location of one gusset, but at the welded connection of the legs to the footplate. After approximately 152,000 load cycles, the loading was stopped, since the cracks had a considerable length of approximately $a=100 \mathrm{~mm}$ each (Figure 4).

The result of the subspace-based damage detection is shown in Fig. 5. The bars represent the mean of 10 to 30 tests executed in one damage state and the error bars represent the related standard deviation. The diagram shows a continuous increase of the $\chi^{2}$-test value, except for the final load series, where a significant drop of the value is noticeable. The reason for this evolution is, that a first fatigue crack has evolved at one leg-footplate connection during load series 3 to 9 . Due to stress redistribution in the last loading series, a second fatigue crack developed at the other leg-footplate connection of the structure. During progression of the first crack, the

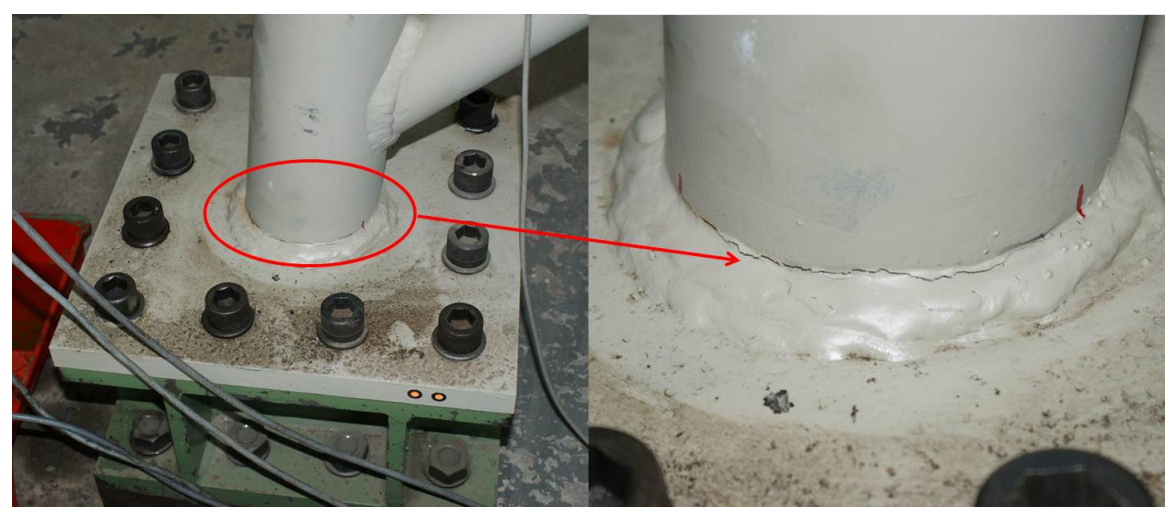

Fig. 4 First fatigue crack at one jacket foot. 


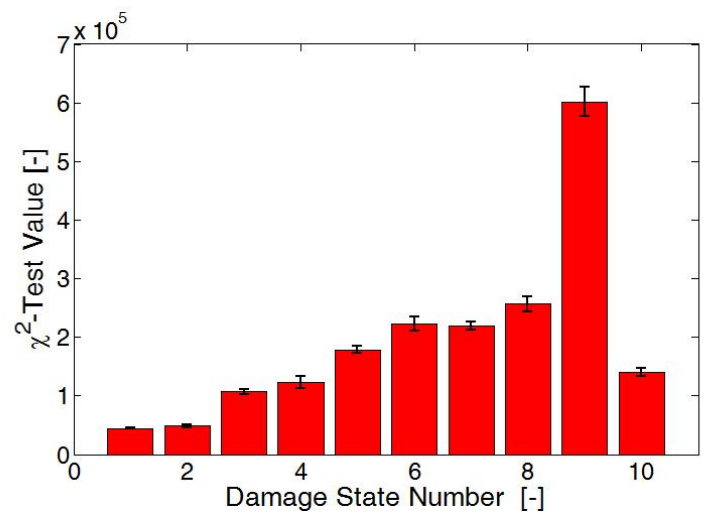

Fig. 5 Mean and standard deviation of damage detection test values for the 10 damage states.

modal system changed gradually with the effect of a continuous increase of the damage test value, while the second crack resulted in a distinct switch back of the modal system and thus led to a decrease of the test value. A detailed description of the fatigue test and discussion of its result is given in [17]. Note that the fatigue damage was successfully detected with the presented technique. The relationship between the test value and the actual damage extent is non-trivial and requires the usage of further damage quantification techniques [14].

\section{Application to S101 Bridge in progressive damage test}

Within the European research project, "Integrated European Industrial Risk Reduction System (IRIS)" a prestressed concrete bridge was artificially damaged [23]. The intention was to provide a complete set of monitoring data during a defined loss of structural integrity for testing and evaluation of various SHM methods and applications. Therefore, the static and dynamic behavior of the structure was measured permanently during the three-day damaging process. The progressive damage campaign was planned and organized by the Austrian company VCE. The characteristics of the structure under observation, the measurement campaign and the introduced damages are presented briefly.

\subsection{The S101 bridge}

The S101 Bridge was a prestressed concrete bridge from the early 1960s and, therefore, a characteristic representative of the partly invalid highway infrastructure asset in Europe. Despite a general lack of experience in the time of their design, pre- 
stressed concrete was a very popular construction type in those days. Retrospectively, some of the major design assumptions proofed to be erroneous and after short periods of operation significant and characteristic damage patterns occurred at the structure. The load bearing capacity and especially the durability of the bridges remained mostly limited despite costly retrofitting activities. In addition, an increase of heavy load vehicle traffic has been ongoing since the start of operation. Thus, prestressed bridge structures in central Europe have been exposed to degradation processes initiated by poor design, while the operational loading and the associated dynamic stressing increases steadily in the same time.

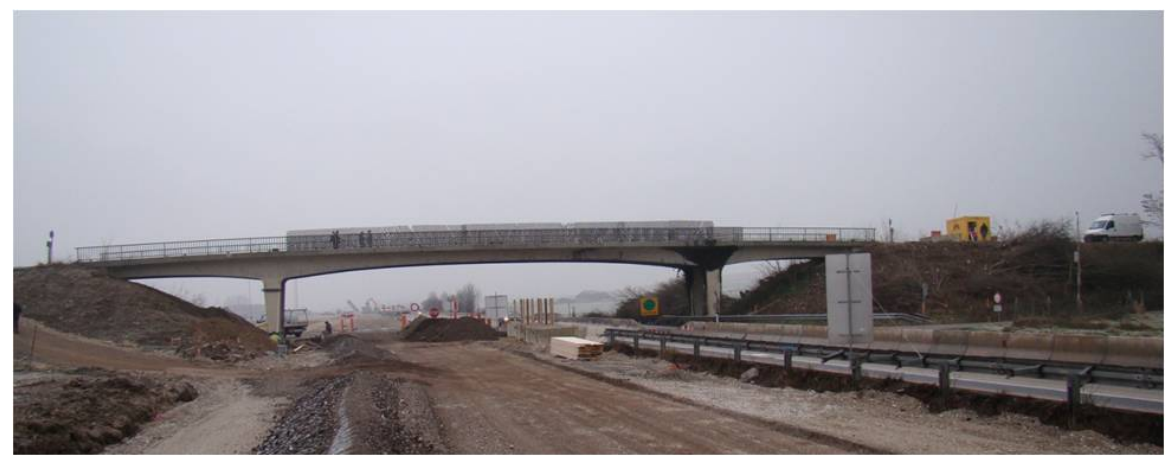

Fig. 6 Bridge S101 during damage test [23].

\subsection{Damage description}

The progressive damage test took place from 10-13 December 2008. During the test the highway beneath the bridge was open in one direction. The second direction was closed for traffic because of construction works, which in addition took place near the bridge.

Two major damage scenarios were artificially induced. First, a significant damage of one of the four columns was inserted by cutting through the column on its lower end. With this action a change in the global structural system was implemented. After a second cut a $5 \mathrm{~cm}$ thick slice of the column was removed and the column was lowered for altogether $3 \mathrm{~cm}$. Afterwards the column was uplifted again to its original position and secured there by steel plates. Then, prestressing tendons of one of the beams were cut successively for a second damage scenario. All in all three and a quarter of a wire bundle were cut through. Between each intersection pauses of several hours were kept to let the structural system change into a new state of equilibrium. Pictures of both scenarios of the progressive damaging are shown in Figure 7. In Table 1 all damage actions are sorted in chronological order. 

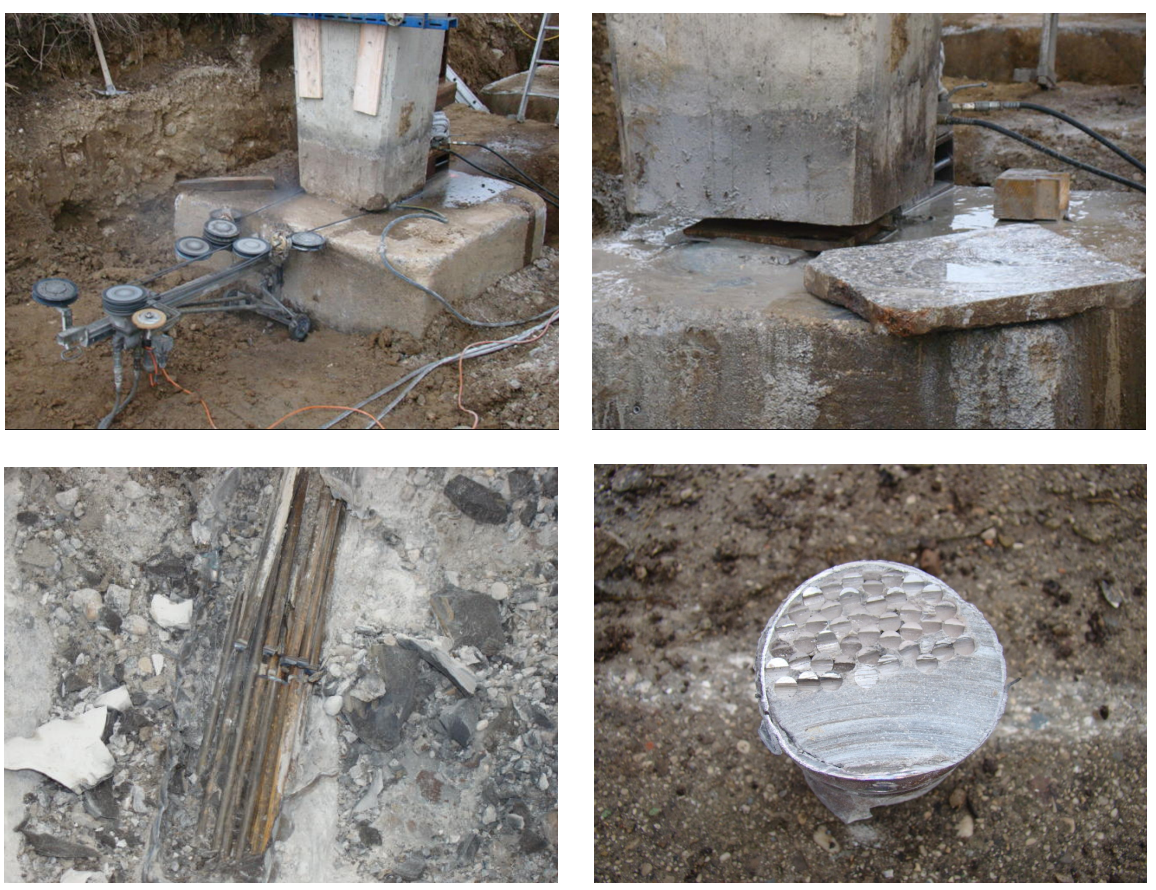

Fig. 7 Progressive damage of column and tendons on bridge S101 [23].

Table 1 Notation of consecutive damage actions.

\begin{tabular}{ll|ll}
\hline \multicolumn{1}{c|}{ Damage Action } & & \multicolumn{1}{c}{ Damage Action } \\
\hline A & Begin of cutting through column & G & Uplifting column \\
B & End of second cut through column & H & Exposing cables and cutting of 1st cable \\
C & Lowering of column -1 st step $(10 \mathrm{~mm})$ & I & Cutting through 2nd cable \\
D & Lowering of column - 2nd step $(20 \mathrm{~mm})$ & J & Cutting through 3rd cable \\
E & Lowering of column - 3rd step $(27 \mathrm{~mm})$ & K & Partly cutting of 4th cable \\
F & Inserting steel plates & & \\
\hline
\end{tabular}

\subsection{Measurement Description}

For the vibration measurements a BRIMOS ${ }^{\circledR}$ measurement system with a permanent sensor grid was used. The grid consisted of 15 sensor locations on the bridge deck (see Fig. 8), in each location three sensors for measurements in the bridge deck's vertical, longitudinal and transversal direction. Altogether, 45 acceleration sensors were applied.

The data were recorded permanently with a sampling frequency of $500 \mathrm{~Hz}$. During the three days measurement campaign, 714 data files each containing 45 chan- 
nels with 165000 data points were produced, corresponding to $5.5 \mathrm{~min}$ of measurements each.

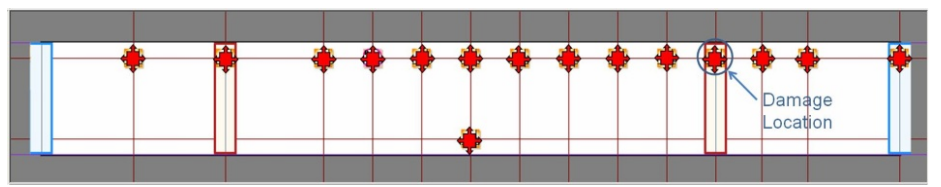

Fig. 8 Location of the sensors on the bridge deck [23].

\subsection{Data analysis}

The vibration measurements were recorded throughout the whole three day long damage test, including the nights. The measurement campaign started approximately 12 hours before the damage of the column was introduced to monitor the undamaged state for an adequate time period.

The reference state of the undamaged structure was set up by computing and averaging the reference matrices from several datasets. With this approach [1], a possible disturbance by single excitation events or different environmental conditions is minimized. In the test stage, the test statistics $s$ in (7) is computed for every data set, which in real time means an indicator of damage for every $5.5 \mathrm{~min}$. Note that no modal parameter estimation or tracking is necessary for this damage detection approach.

\subsection{Results of damage detection}

Figure 9 shows a bar plot of values as damage indicators of all consecutive tests within the three days campaign, where all 45 sensors $\left(r=r_{0}=45\right)$ were used. For the computation of the residual covariance matrix 100 datasets of the undamaged state were used. The abscissa of the plot describes the chronological sequence of the damage activities as noted in Table 1 as well as the 6:00 am and 6:00 pm points of time for orientation.

The reaction of the damage detection test at the main damage events can be easily observed. From the measurements in the reference state (before damage event A), a threshold of the damage indicator can be set easily, such that all the subsequent scenarios after damage event $\mathrm{B}$ would be correctly classified as damaged. This qualifies the proposed method for damage detection. In the following, the obtained results are analyzed in more detail. It can be seen that the damage indication is interfered by noise in the ambient excitation of the bridge. The $s$ values periodically swell up in 


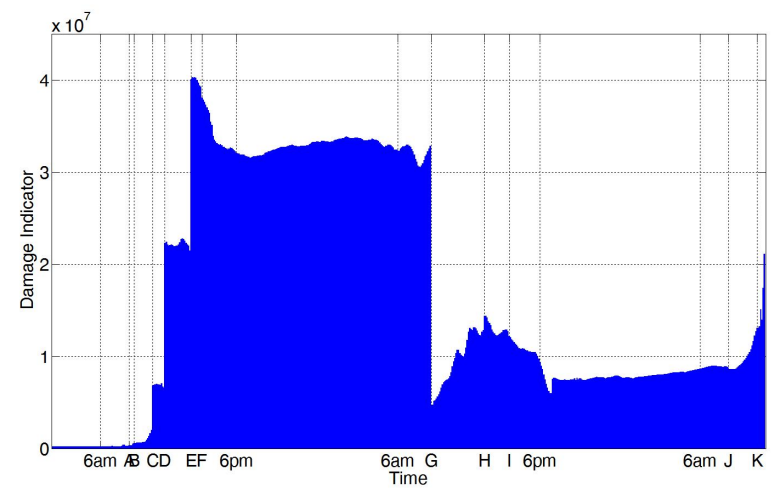

Fig. 9 Course of the damage indicator over the three-day damage test.

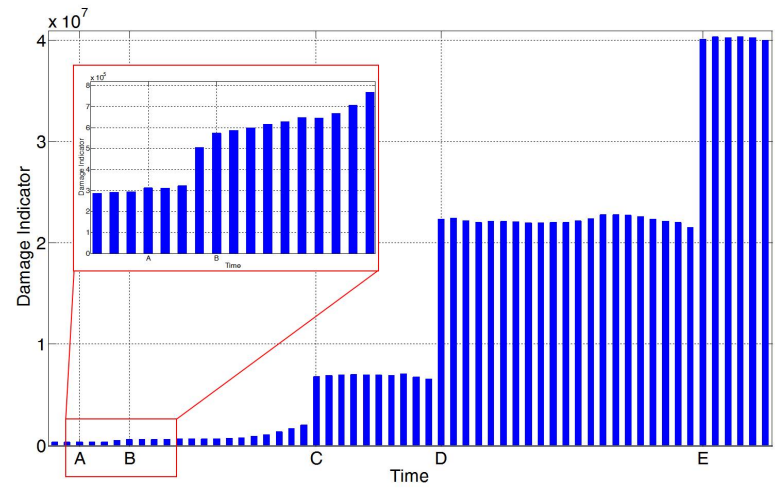

Fig. 10 Damage indicator for cutting and settling of one bridge column, detail of damage indicator for cutting through one bridge column.

the morning and ebb away in the evening. It is assumed that the traffic going underneath the bridge and/or the construction work nearby are the source of the noise. Since the measurements in the reference state were only taken during 12 hours in the night, an extension of the measurement time period of the undamaged state to a whole day circle might reduce these disturbances significantly when taken into account in the residual covariance matrix. Influences by solar radiation and/or temperature alternation can be excluded, since during the three-day campaign misty winter weather with only moderate temperature changes just below freezing was dominant.

Figure 10 shows the damage indicator during the several steps of the first damage scenario, the cutting and settling of one of the four bridge columns. With exception of the time periods of the direct mechanical destruction processes, the displayed sequence of damage indicators has a consecutive course. As can be seen in Fig. 10, the three steps of the column settlement action are very distinctive in their influence 


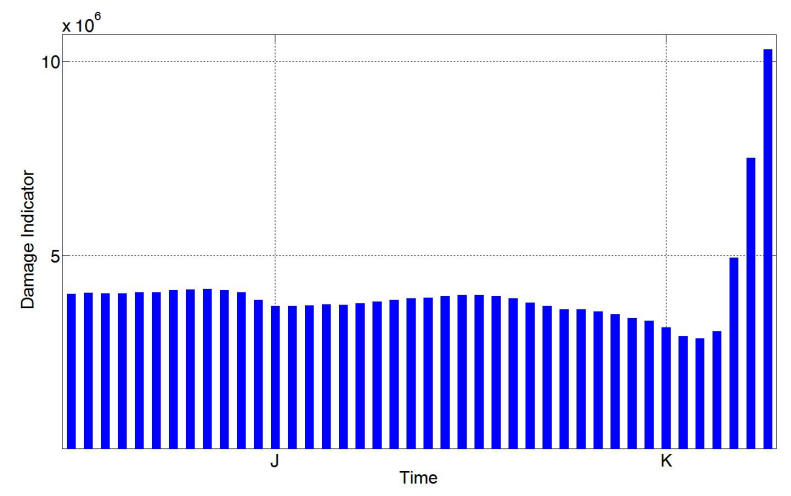

Fig. 11 Detail of damage indicator at the end of the tendon cutting process.

on the computed damage indicator. Obviously, the dynamic system has changed to quite some extent, and the elastic settlement of altogether $27 \mathrm{~mm}$ can be clearly detected. Although not that strongly visible, the cutting of the column $(A+B)$ also caused an increase of the indicator of approximately $75 \%$. However it has to be mentioned that the absolute effect is superimposed by noise effects. The inlet in Fig. 10 shows a detail of that time period.

The column remained in the settled condition for approximately one day and was then uplifted again in its former position (event G). The effect of the uplifting is again clearly visible in Fig. 9 by a drop of the damage indicator. However, the indicator did not drop completely to its original value, which is certainly due to the fact that the lowering of one column has led to cracking within the concrete structure to some extent and hence to a change of the dynamic signature of the system.

As one can see in Fig. 9, the cutting of the prestressing tendons did not lead to a significant change in the damage indicator after the single cutting steps. Nevertheless, a distinctive increase of the indicator could be observed at the end of the measurement. Figure 11 shows the last time period in detail. It is possible that a change of the bridge's structural system took place with a time delay after cutting partly the fourth tendon.

For a prestressed concrete structure, the loss of prestressing is a major damage that comes along with a significant loss of its load bearing capacity. One reason that the cutting of the tendons does not affect the proposed damage indicator might be that the overall prestressing is designed for combinations of dead and traffic loads. Since the bridge deck of S101 has a quite slender cross section, the dead load is not that high in comparison with the maximum design traffic loading. An additional dead load reduction comes from the removal of the asphalt surface before the damage test. Furthermore it has to be recalled that the prestressing was designed with adequate safety margins. Also, the cutting results only in local loss of prestressing because of the bound between tendons and concrete. For these specified reasons it is assumed that the cutting of the tendons during the damage test did not lead to a sig- 
nificant change of the global structural system, since the loading of the structure was not high enough to activate the damage right after its insertion. The increase of the indicator at the end of the test series might be the result of a delayed rearrangement of the structural system by a reduction of stresses under generation of cracks in the concrete of the bridge deck. Though, an evidence of that assumption, for instance by an increase of the measured bridge deck deflection, could not be found.

\section{Conclusions}

In this chapter, a method for vibration-based damage detection has been presented that combines statistical robustness with simplicity of application. A damage test statistic is computed entirely from measurement data of a (healthy) reference system and the current system, without the need of a finite element model. The method is designed for the detection of small damages before they grow to dangerous extents, while taking the intrinsic uncertainties of vibration measurements due to unknown excitation and noise into account. The damage detection method has been validated successfully on a laboratory test case and on a full scale damage test on a real highway bridge. Thanks to its properties, it is well suited for being embedded in automated structural health monitoring systems. Extensions of the damage detection method to damage localization and quantification in the same framework [3,14] are possible in connection with a finite element model, being a step towards the development of robust damage assessment systems that will combine measurement data with physical models in the future.

Acknowledgements We thank Dr. Helmut Wenzel, VCE, and the FP7 IRIS project for providing the data from S101 Bridge.

\section{Appendix}

The damage detection test statistic $s$ is set up using measurements from the reference state of the structure, requiring the estimation of the left null space matrix $S$, the residual covariance matrix $\Sigma$ and setting up a threshold $t$.

First, the Hankel matrix $\widehat{\mathscr{H}}_{p+1, q}^{0}$ is computed from the reference measurements. Then, the left null space matrix $S$ can be estimated from its SVD

$$
\widehat{\mathscr{H}}_{p+1, q}^{0}=\left[\begin{array}{ll}
U_{1} & U_{0}
\end{array}\right]\left[\begin{array}{cc}
\Delta_{1} & 0 \\
0 & \Delta_{0}
\end{array}\right]\left[\begin{array}{l}
V_{1} \\
V_{0}
\end{array}\right]
$$

as $\widehat{S}=U_{0}$, where the SVD is truncated at the desired model order $n$ with $\Delta_{1} \in \mathbb{R}^{n \times n}$ and $\Delta_{0} \approx 0$. 
Second, the residual covariance matrix $\Sigma$ is obtained by separating the available reference dataset into $n_{b}$ data blocks of length $N_{b}$, such that the total data length yields $N=n_{b} N_{b}$. On each block, the Hankel matrix is computed as

$$
\widehat{R}_{i}^{(j)}=\frac{1}{N_{b}} \sum_{k=1+(j-1) N_{b}}^{j N_{b}} y_{k} y_{k-i}^{T}, \quad \widehat{\mathscr{H}}_{p+1, q}^{(j)}=\operatorname{Hank}\left(\widehat{R}_{i}^{(j)}\right)
$$

Then, $\widehat{\mathscr{H}}_{p+1, q}^{0}=\frac{1}{n_{b}} \sum_{j=1}^{n_{b}} \widehat{\mathscr{H}}_{p+1, q}^{(j)}$ and the covariance estimate of the residual follows from the covariance of the sample mean as

$$
\widehat{\Sigma}=\frac{N_{b}}{n_{b}-1} \sum_{j=1}^{n_{b}} \operatorname{vec}\left(S^{T} \widehat{\mathscr{H}}_{p+1, q}^{(j)}-S^{T} \widehat{\mathscr{H}}_{p+1, q}^{0}\right) \operatorname{vec}\left(S^{T} \widehat{\mathscr{H}}_{p+1, q}^{(j)}-S^{T} \widehat{\mathscr{H}}_{p+1, q}^{0}\right)^{T}
$$

Finally, compute the test statistic $s$ for several training datasets of length $N$ from the reference state, and determine the threshold $t$ from the test values for a desired type I error.

\section{References}

1. Balmès, E., Basseville, M., Bourquin, F., Mevel, L., Nasser, H., Treyssède, F.: Merging sensor data from multiple temperature scenarios for vibration-based monitoring of civil structures. Structural Health Monitoring 7(2), 129-142 (2008)

2. Balmès, E., Basseville, M., Mevel, L., Nasser, H.: Handling the temperature effect in vibrationbased monitoring of civil structures: a combined subspace-based and nuisance rejection approach. Control Engineering Practice 17(1), 80-87 (2009)

3. Balmès, E., Basseville, M., Mevel, L., Nasser, H., Zhou, W.: Statistical model-based damage localization: a combined subspace-based and substructuring approach. Structural Control and Health Monitoring 15(6), 857-875 (2008)

4. Basseville, M., Abdelghani, M., Benveniste, A.: Subspace-based fault detection algorithms for vibration monitoring. Automatica 36(1), 101-109 (2000)

5. Basseville, M., Bourquin, F., Mevel, L., Nasser, H., Treyssède, F.: Handling the temperature effect in vibration monitoring: two subspace-based analytical approaches. Journal of Engineering Mechanics 136(3), 367-378 (2010)

6. Basseville, M., Mevel, L., Goursat, M.: Statistical model-based damage detection and localization: subspace-based residuals and damage-to-noise sensitivity ratios. Journal of Sound and Vibration 275(3), 769-794 (2004)

7. Bernal, D.: Kalman filter damage detection in the presence of changing process and measurement noise. Mechanical Systems and Signal Processing 39(1-2), 361-371 (2013)

8. Brownjohn, J., De Stefano, A., Xu, Y., Wenzel, H., Aktan, A.: Vibration-based monitoring of civil infrastructure: challenges and successes. Journal of Civil Structural Health Monitoring 1(3), 79-95 (2011)

9. Carden, E., Fanning, P.: Vibration based condition monitoring: a review. Structural Health Monitoring 3(4), 355-377 (2004)

10. Döhler, M., Hille, F.: Subspace-based damage detection on steel frame structure under changing excitation. In: Proc. 32nd International Modal Analysis Conference. Orlando, FL, USA (2014) 
11. Döhler, M., Hille, F., Mevel, L., Rücker, W.: Structural health monitoring with statistical methods during progressive damage test of S101 Bridge. Engineering Structures 69, 183-193 (2014)

12. Döhler, M., Mevel, L.: Subspace-based fault detection robust to changes in the noise covariances. Automatica 49(9), 2734-2743 (2013)

13. Döhler, M., Mevel, L., Hille, F.: Subspace-based damage detection under changes in the ambient excitation statistics. Mechanical Systems and Signal Processing 45(1), 207-224 (2014)

14. Döhler, M., Mevel, L., Zhang, Q.: Fault detection, isolation and quantification from Gaussian residuals with application to structural damage diagnosis. Annual Reviews in Control 42, 244-256 (2016)

15. Fan, W., Qiao, P.: Vibration-based damage identification methods: a review and comparative study. Structural Health Monitoring 10(1), 83-111 (2011)

16. Farrar, C., Worden, K.: An introduction to structural health monitoring. Philosophical Transactions of the Royal Society A: Mathematical, Physical and Engineering Sciences 365(1851), 303-315 (2007)

17. Hille, F., Petryna, Y., Rücker, W.: Subspace-based detection of fatigue damage on a steel frame laboratory structure for offshore applications. In: Proceedings of the 9th International Conference on Structural Dynamics, EURODYN 2014. Porto, Portugal, July 2014 (2014)

18. Juang, J.N.: Applied system identification. Prentice Hall, Englewood Cliffs, NJ, USA (1994)

19. Kullaa, J.: Damage detection of the Z24 Bridge using control charts. Mechanical Systems and Signal Processing 17(1), 163-170 (2003)

20. Ramos, L., Marques, L., Lourenço, P., De Roeck, G., Campos-Costa, A., Roque, J.: Monitoring historical masonry structures with operational modal analysis: Two case studies. Mechanical Systems and Signal Processing 24(5), 1291-1305 (2010)

21. Rytter, A.: Vibrational based inspection of civil engineering structures. Ph.D. thesis, Aalborg University, Denmark (1993)

22. Structural Vibration Solutions A/S: ARTeMIS Modal Pro - Damage Detection Plugin (2015). http://www.svibs.com

23. VCE: Progressive damage test S101 Flyover Reibersdorf / draft. Tech. Rep. 08/2308, VCE (2009)

24. Worden, K., Manson, G., Fieller, N.: Damage detection using outlier analysis. Journal of Sound and Vibration 229(3), 647-667 (2000)

25. Yan, A., De Boe, P., Golinval, J.: Structural damage diagnosis by Kalman model based on stochastic subspace identification. Structural Health Monitoring 3(2), 103-119 (2004) 